\title{
Systematic construction of common channel hopping rendezvous strategies in cognitive radio networks
}

\author{
Erik Ortiz Guerra', Vitalio Alfonso Reguera' ${ }^{1}$, Richard D Souza ${ }^{2}{ }^{*}$, Evelio G Fernández ${ }^{3}$ and Marcelo E Pellenz ${ }^{4}$
}

\begin{abstract}
Cognitive radio networks (CRNs) have emerged as a promising paradigm that can solve the shortage of spectrum resources, providing the ability to adapt and opportunistically exploit the spectrum holes. The rendezvous process allows cognitive users to find a common channel and establish a communication link. In this paper, we focus on the design of fast blind rendezvous algorithms to guarantee that every node should be able to rendezvous in all common available channels. We follow a systematic approach by first proposing a role-based algorithm that ensures maximum rendezvous diversity and then extending it to a common strategy through the use of multiples radios. Both proposals guarantee rendezvous under symmetric and asymmetric models. Simulation results show that our proposals outperform other recently developed rendezvous protocols with similar approaches in terms of expected time to rendezvous and maximum time to rendezvous.
\end{abstract}

Keywords: Cognitive radio; Blind rendezvous; Rendezvous diversity; Channel hopping sequences

\section{Introduction}

Recent spectrum measurements indicate that much of licensed spectrum is underutilized most of the time and unlicensed spectrum has been overcrowded due to the growth of wireless applications. Cognitive radio networks (CRNs) have been proposed to solve the shortage of usable spectrum and improve the efficiency in the usage of licensed spectrum $[1,2]$.

In CRNs, each cognitive device, or secondary user (SU), has one or more radios capable of sensing the spectrum and detecting portions that are not being used by licensed users, or primary users (PU). To establish a communication link, two SUs should meet on a common channel and exchange handshake information, this process is referred to as rendezvous and is essential in the CRNs operation [3]. Because of the different locations of SUs, the set of spectrum holes, also called available channel set, may not be the same for each SU. If the set of available channels is the same for all SUs, then we are dealing with a symmetric

\footnotetext{
${ }^{*}$ Correspondence: richard@utfpr.edu.br

${ }^{2}$ Federal University of Technology, Paraná (UTFPR), Avenida Sete de Setembro, Curitiba, Brazil

Full list of author information is available at the end of the article
}

model; otherwise, the model is said to be asymmetric (i.e., different SUs perceive different available channels).

A simple way to solve the rendezvous problem is by using a common control channel (CCC) [4]. In this approach, one of the available channels is assigned as the CCC and serves as the rendezvous channel. Some works have proposed static and dynamic CCC selection [5-10]. Although they have good theoretical performance, their main drawback is that a CCC constitutes a single point of failure, experiencing congestion when the number of PUs increases and facing the possibility that the CCC is occupied by a PU $[11,12]$. Another approach is to use channel hopping $(\mathrm{CH})$ sequences, in which each $\mathrm{SU}$ visits the network channels following a predefined strategy in order to achieve rendezvous in any of the available channels. This process is referred to as blind rendezvous and overcomes the drawbacks of CCC.

Additionally, according to the SUs' behavior, there are different types of rendezvous strategies [13]. If SUs have preassigned roles (e.g., transmitter or receiver), they can follow different algorithms to generate their $\mathrm{CH}$ sequences and perform a role-based (RB) strategy. These strategies have been proven to be optimal in minimizing the time to rendezvous, and the sequences generated by

\section{它 Springer}

(c) 2015 Guerra et al: licensee Springer. This is an Open Access article distributed under the terms of the Creative Commons Attribution License (http://creativecommons.org/licenses/by/4.0), which permits unrestricted use, distribution, and reproduction in any medium, provided the original work is properly credited. 
each SU are different. On the other hand, the preassigned role is not possible in some scenarios, and in these cases, the SUs must perform a common strategy (CS) to generate their $\mathrm{CH}$ sequences. Unlike the RB strategy, in CS the sequences generated by each SU are identical. Mixed or hybrid strategies are also possible in which the SUs use the same algorithm to generate the $\mathrm{CH}$ sequences but the generated sequences are different (e.g., by using a random seed). Furthermore, CS-CH sequences can be deterministic, in which case they are periodically repeated, or can be generated following a random pattern. The latter approach has been used in [12,14]. The authors in [12] show that in scenarios with intermittent PU activity the use of deterministic sequences offers no advantages over random $\mathrm{CH}$ algorithms. However, the main drawback of the random solutions is that they cannot guarantee the rendezvous in finite time under any circumstance. The analysis of random sequences is out of the scope of this paper, whose main focus is on the design of deterministic sequences.

Most works regarding $\mathrm{CH}$ rendezvous [14-29] consider that the time is divided into slots and the time to rendezvous (TTR) is defined as the number of time slots needed to successfully rendezvous after all SUs have begun the $\mathrm{CH}$ sequences. Moreover, the expected TTR (ETTR) and the maximum TTR (MTTR) are two important metrics commonly used to evaluate these strategies and are often computed when all channels are available. The authors in $[15,16]$ adopt the term maximum conditional TTR (MCTTR) to refer to the MTTR when not all channels are available to all SUs. Some authors $[15,17,27,30]$ also consider in the design of $\mathrm{CH}$ sequences the number of channels in which the rendezvous may occur (rendezvous diversity). However, most of them only make a qualitative analysis of this parameter. A metric for evaluating the rendezvous diversity can be found in $[15,19,21]$. As expected, in the asymmetric model, the algorithms with greater rendezvous diversity perform better than the mechanisms where the rendezvous is only achieved in a few channels.

In this paper, we focus on the design of deterministic $\mathrm{CH}$ sequences based algorithms to solve the blind rendezvous problem. Our main purpose is to develop a fast rendezvous algorithm which guarantees that SUs may meet in all available channels. We follow a systematic approach by first proposing a full diversity channel hopping $(\mathrm{FDCH})$ role-based algorithm that ensures maximum rendezvous diversity, and then extending it to a common strategy through the use of multiples radios. We include a theoretical analysis as well as extensive simulations for two-user and multi-user CRNs under symmetric and asymmetric models, in order to demonstrate that the proposed strategies outperform other recently proposed ones.
The remainder of this paper is organized as follows. Related work is reviewed in Section 2. The system model and problem formulation is presented in Section 3. In Section 5, we propose the FDCH role-based (FDCH-RB) and $\mathrm{FDCH}$ common strategy (FDCH-CS) algorithms and present their theoretical performance analysis. Section 6 extends our proposal to the multi-user scenario. Simulation results are presented in Section 7. Finally, Section 8 concludes the paper.

\section{Related works}

Several algorithms have been proposed to solve the blind rendezvous issue. In [17], the asynchronous channel hopping $(\mathrm{ACH})$ algorithm is proposed where the authors focus on designing $\mathrm{CH}$ sequences that ensure rendezvous in all available channels and use the term rendezvous diversity to denote the number of channels in which the rendezvous may occur. First, they present a transmitter and a receiver sequence (i.e., a RB strategy) and then propose a CS strategy that allows each SU to alternate between these sequences, as to guarantee rendezvous and ensure maximum rendezvous diversity (i.e., rendezvous in all available channels). Recently, in [18], we proposed a simple role-based (SRB) strategy for the symmetric model that outperforms the $\mathrm{ACH}(\mathrm{RB})$ algorithm. However, the $\mathrm{SRB}$ is unable to guarantee rendezvous in the asymmetric model.

The authors in [15] present four quorum-based channel hopping $(\mathrm{QCH})$ strategies. These algorithms are role based. The M-QCH and L-QCH are described under the assumption of global time synchronization between SUs (i.e., synchronous environment), while A-QCH and A$\mathrm{MOCH}$ do not need time synchronization between SUs. Although A-QCH and A-MOCH work in asynchronous environments, the $\mathrm{A}-\mathrm{MOCH}$ ensures maximum rendezvous diversity while A-QCH does not. Like $\mathrm{ACH}(\mathrm{RB})$ and $\mathrm{A}-\mathrm{MOCH}$, our approach considers a role-based strategy which ensures maximum rendezvous diversity but with a different sequence construction scheme that guarantees rendezvous in both, synchronous and asynchronous, environments. Also we extend our proposal to a common strategy by the use of two radios.

Two RB strategies called rendezvous couple channel hopping $(\mathrm{RCCH})$ and asynchronous rendezvous channel hopping $(\mathrm{ARCH})$ are proposed in [19]. The first one considers that the SUs have global clock synchronization while the other assumes an asynchronous scenario and both ensure maximum rendezvous diversity when the number of network channels is even. Also this paper presents the symmetric asynchronous rendezvous channel hopping (SARCH) which is a CS strategy but not ensures maximum diversity. In our proposal, no global clock synchronization is assumed, neither the number of network channels is constrained. 
As in $\mathrm{ACH}, \mathrm{A}-\mathrm{MOCH}, \mathrm{RCCH}$, and $\mathrm{ARCH}$ algorithms, the authors in $[20,21]$ consider the rendezvous diversity in the design of an efficient channel hopping (ETCH) algorithm. This algorithm has two variants; first, the authors propose SYNC-ETCH which considers synchronous environments. Next, the ASYN-ETCH algorithm is proposed which does not assume the existence of global clock synchronization. Both ensure maximum rendezvous diversity. The main drawback of the ASYN-ETCH is that it exhibits a greater MTTR than that of other similar proposals [19].

In [22] three algorithms called generated orthogonal sequence (GOS) based, modular clock (MC), and modified modular clock (MMC) are presented. The GOS and MC algorithms only work under the symmetric model while MMC provides asymmetric model support. In terms of ETTR, the MC and MMC performance is better than that of GOS, but they do not guarantee rendezvous.

Other $\mathrm{CS}-\mathrm{CH}$ sequence algorithms are introduced in [23] and [24]: the deterministic rendezvous (DRSEQ) and the channel rendezvous (CRSEQ). Although both schemes guarantee rendezvous, none of them guarantees maximum rendezvous diversity. The DRSEQ algorithm only works under the symmetric model and outperforms GOS, MC, and CRSEQ in this scenario.

The aforementioned algorithms consider that the SUs follow a $\mathrm{CH}$ sequence for visiting each of the channels in the network and once in the selected channel perform spectrum sensing to identify whether the channel is free of PU activity. This strategy can be effective when the channels used by the PUs change dynamically. However, in scenarios where these channel changes do not occur or occur very slowly, other mechanisms are more suitable. In this paper, we consider that during the rendezvous attempt the PUs operate in a fixed set of channels and that the SUs have prior knowledge of the state of each of the network channels ${ }^{\mathrm{a}}$. With this knowledge, some authors [16,25-29] provide each SU with the ability to attempt to rendezvous only in a channel previously identified as available (free of PU interference).

In [25] the ring walk (RW) rendezvous algorithm and two variants, RW1 and RW2, are proposed. Both work under symmetric and asymmetric models, guarantee multi-user rendezvous, and outperform GOS, MC, and MMC in terms of ETTR. The performance of RW2 is better than RW1, but none of them ensures maximum rendezvous diversity.

The authors in [26] propose the jump stay (JS) algorithm which guarantees rendezvous under symmetric and asymmetric models, supports multi-user rendezvous, and ensures maximum rendezvous diversity. In [27] the same authors report the enhanced jump stay (EJS) algorithm that also guarantees rendezvous in all available channels and improves over JS in terms of ETTR and MTTR for the asymmetric model but slightly increases the MTTR in the symmetric model. The fast rendezvous channel hopping $(\mathrm{FRCH})$ algorithm is introduced in [16] and is shown to outperform JS in terms of ETTR. In [28] we propose the short sequence-based (SSB) rendezvous algorithm which outperforms the FRCH and EJS algorithm in terms of ETTR and MTTR but, like FRCH, does not guarantee rendezvous in all available channels.

Except in the $\mathrm{ACH}(\mathrm{RB}), \mathrm{SRB}, \mathrm{RCCH}$, and $\mathrm{ARCH}$ in all other protocols described above, all SUs use the same algorithm to generate the $\mathrm{CH}$ sequences. However, in the SYNC-ETCH, ASYN-ETCH, MC, MMC, JS, EJS, and RW algorithms, each $\mathrm{SU}$ uses different parameters to generate its $\mathrm{CH}$ sequence. Thus, even when using a common algorithm, the jump sequences generated by each SU are different. These algorithms are an hybrid variant between CS and RB strategies. Note that they do not need preassigned roles, but the hopping sequences are different for each SU. One way to generate different $\mathrm{CH}$ sequences is that each SU uses random selected parameters, as this is the approach used in the MC, MMC, JS, and EJS algorithms. On the other hand, in the RW algorithm, each SU uses its ID as a seed to generate the $\mathrm{CH}$ sequences.

Most of the reported algorithms [16-28] implicitly consider that each $\mathrm{SU}$ is equipped with one radio. However, the use of multiple radios can help to reduce the TTR. This view is presented in [29] where a role-based parallel sequence (RPS) is proposed to exploit multiple radios and reduce the ETTR in a two-user scenario. The SUs use an hybrid strategy to generate the $\mathrm{CH}$ sequences which ensure rendezvous and maximum rendezvous diversity. The use of multiple radios allows each radio to use a different $\mathrm{CH}$ sequence, so that each $\mathrm{SU}$ assumes more than one role simultaneously. Our approach is similar to that described in [29], but with different $\mathrm{CH}$ sequences, providing better performance in most practical scenarios as can be seen in Section 7.

Table 1 shows a brief comparison of some of the above mentioned algorithms considering the asymmetric model support, rendezvous diversity, and rendezvous strategy. Also we include the theoretical results in terms of ETTR, MTTR, and MCTTR as provided by the respective authors.

\section{System model and problem formulation}

We consider a CRN consisting of $M \geq 2$ SUs which coexist with some PUs in the same geographical area. The PUs are the licensed users of the spectrum, which can be divided into $N \geq 2$ non-overlapping channels, labeled as $0,1, \ldots N-1$, and these labels are the same for all SUs. We assume that the time is divided into slots of equal duration. For any time slot $t \in \mathbb{N}^{\mathrm{b}}$ let $S_{u}(t)$, $S_{u}: \mathbb{N}^{*} \rightarrow\{0, \ldots, N-1\}$, be the $\mathrm{CH}$ sequence for $\mathrm{SU}$ 
Table 1 Summary of representative rendezvous protocols

\begin{tabular}{|c|c|c|c|c|c|c|}
\hline Protocol & ETTR & MTTR & MCTTR & $\begin{array}{c}\text { Asymmetric } \\
\text { model support }\end{array}$ & $\begin{array}{l}\text { Maximum } \\
\text { rendezvous diversity }\end{array}$ & Strategy \\
\hline $\mathrm{ACH}(\mathrm{RB})[17]$ & Unknown & Unknown & Unknown & $\checkmark$ & $\checkmark$ & $\mathrm{RB}$ \\
\hline A-MOCH [15] & Unknown & $N^{2}-N+1$ & $N^{2}$ & $\checkmark$ & $\checkmark$ & $\mathrm{RB}$ \\
\hline SRB [18] & $\frac{N-1}{2}$ & $N-1$ & Unknown & & & $\mathrm{RB}$ \\
\hline $\mathrm{ARCH}[19]$ & Unknown & $2 N-1^{\star}$ & $N^{2 \star}$ & $\checkmark$ & $\checkmark^{\star}$ & $\mathrm{RB}$ \\
\hline ASYN-ETCH [21] & $\frac{2 N^{2}+N}{N-1}$ & Unknown & Unknown & $\checkmark$ & $\checkmark$ & Hybrid \\
\hline GOS [22] & $\frac{N^{4}+2 N^{2}+6 N-3}{3 N^{2}+3 N}$ & $N(N+1)$ & Unknown & & & $\mathrm{CS}$ \\
\hline MC [22] & $\frac{2 P^{2}}{P-1}$ & Unknown & Unknown & & & Hybrid \\
\hline MMC [22] & Unknown & Unknown & Unknown & $\checkmark$ & & Hybrid \\
\hline DRSEQ [23] & Unknown & $2 N+1$ & Unknown & & & CS \\
\hline CRSEQ [24] & Unknown & Unknown & $P(3 P-1)$ & $\checkmark$ & & CS \\
\hline RW2 [25] & $(N-1)\left(\ln ^{n}+\frac{1}{2}\right)$ & $n(N-1)$ & $2 n(N+1-G)(N-1)$ & $\checkmark$ & & Hybrid \\
\hline JS [26] & $\frac{5 P}{3}+3$ & $3 P$ & $3 N P(P-G)+3 P$ & $\checkmark$ & $\checkmark$ & Hybrid \\
\hline EJS [27] & $\frac{3 P}{2}+3$ & $4 P$ & $4 P(P+1-G)$ & $\checkmark$ & $\checkmark$ & Hybrid \\
\hline $\mathrm{FRCH}[16]$ & Unknown & $2 N+1$ & $N(2 N+1)^{\star \star}$ & $\checkmark$ & & CS \\
\hline SSB [28] & $\frac{2(N-1)^{2}}{2 N-1}$ & $2 N-2$ & $(N-1)(2 N-1)$ & $\checkmark$ & & $\mathrm{CS}$ \\
\hline $\mathrm{RPS}^{\star \star \star}[29]$ & (1) & $(2)$ & Unknown & $\checkmark$ & $\checkmark$ & Hybrid \\
\hline
\end{tabular}

$N$, number of available channels. $P$, smallest prime greater or equal than $N$ for MC and CRSEQ, but $N+1$ for JS and EJS. $n$, number of nodes in the network. $G$, number of common available channels for two-user scenario. ${ }^{\star}$ These results are valid when $N$ is even, please see Table one in [19] for further details. ${ }^{\star \star}$ This expression is only valid for some values of $N$, please see [16] for further details. ${ }^{\star \star \star}$ In the RPS algorithm, each SU is equipped with more than one radio. (1): ETTR $=\left[\frac{P}{\text { max }(u, v)-1}\right]+$ $\frac{\left(\left\lceil\frac{p}{\max (u, v)-1}\right]-1\right)^{2}}{2\left[\frac{p}{\min (u, v)-1}\right]} \cdot(2): \operatorname{MTTR}=\left\lceil 2\left\lceil\frac{p}{\max (u, v)}\right\rceil-1\right\rceil \cdot u, v$, number of radios of SUs attempting to rendezvous. ARCH(RB), asynchronous channel hopping (role based). A-MOCH, asynchronous maximum overlapping channel hopping. SRB, simple role-based. ARCH, asynchronous rendezvous channel hopping. ASYN-ETCH, asynchronous efficient channel hopping. GOS, generated orthogonal sequences. MC, modular clock. MMC, modified modular clock. DRSEQ, deterministic rendezvous sequence. CRSEQ, channel rendezvous sequence. RW2, ring walk 2. JS, jump stay. EJS, enhanced jump stay. FRCH, fast rendezvous channel hopping. SSB, short sequence-based. RPS, role-based parallel sequence.

$u, u \in\{1,2, \ldots M\}$. Let $A_{u} \subseteq\{0,1,2, \ldots, N-1\}$ be the set of available channels for $\mathrm{SU} u$, where a channel is said to be available for SU communication if PU activity is not detected in this channel. Like [16,25-27] we consider that each SU know its set of available channels (i.e., $A_{u}$ ) before the rendezvous process begins. Let $G$ be the number of commonly available channels for all SUs (i.e., $G=\left|\bigcap_{\forall u} A_{u}\right|$, where $\bigcap_{\forall u} A_{u}$ is the intersection between the set of available channels for all SUs and $|\cdot|$ is the cardinality operator). Note that $G \neq 0$ is a necessary condition for a feasible rendezvous solution.

According to the channels' availability, we consider both symmetric and asymmetric models. In the symmetric model, the SUs perceive the same available channels, so that $A_{i}=A_{j}, \forall i, j \leq M$. For the asymmetric model, different SUs might perceive different available channels.

We also assume that the SUs have not global time synchronization and each one has a local independent clock. We emulate slot boundary synchronization following a similar approach as in $[23,26]$. Let $\tau$ be the minimum duration for exchanging the necessary handshake information. In [23] the authors proved that since the SUs may not be time synchronized, the duration of a time slot of at least $2 \tau$ ensures that the overlap of any two slots is sufficient to complete the rendezvous process. As proved in [26], this is equivalent to consider that the SUs are synchronized with respect to slot boundaries. Moreover, we consider that the SUs can start its $\mathrm{CH}$ sequences at different time slots.

We consider important to highlight that even if two SUs are in the same channel, the communication between them can fail due to fading, contention with other SUs or some other situations that affect the quality of the channels. Then, if the $\mathrm{CH}$ sequence only guarantees that SUs meet in few channels, the rendezvous will be conditioned by the state of those channels and eventually will degrade the TTR performance. Thus, in addition to ensuring rendezvous, it is also important to diversify the channels where rendezvous may occur (i.e., to maximize rendezvous diversity). To compute the rendezvous diversity, we introduce a new metric, the rendezvous diversity index, which is a variation of the degree of rendezvous proposed in [19].

Definition 1. In a $C R N$ with $G$ common available channels for SUs, if $C:|C| \leq G$ is a set of channels in which the 
rendezvous occurs, the rendezvous diversity index can be computed as: $D=\frac{|C|}{G}$.

The rendezvous diversity is maximized when $|C|=G$ which minimizes the risk of rendezvous failures. For some rendezvous protocols $[16,22-25,28]$, the cardinality of $C$ is a function of the channels in which each SU starts the rendezvous process. Accordingly, we consider $\bar{D}$ as the mean value of the rendezvous diversity index and when $\bar{D}=1$ we say that the rendezvous scheme ensures maximum rendezvous diversity.

\section{Basic idea for $\mathrm{CH}$ sequence construction}

Similar to $[18,24]$, we allocate $T$ discrete points in a ring, as shown in Figure 1, such that each point represents one of the $N$ network channels. In order to maximize rendezvous diversity, all channels appears at least once in the ring, so that $T \geq N$. Note that this is an abstraction in order to clarify the analysis in the rest of this paper. In the CRN context, two SUs are initially placed in any of the $T$ positions in the ring. In the literature, there are some classical rendezvous algorithms that work over continuous rings, as the OP-DIR algorithm [13], in which those intending to rendezvous jump in opposite directions over the ring. However, since the SUs jump at discrete positions in the CRN context, the use of such classical rendezvous algorithm is not effective in all cases. Next, some considerations regarding this issue are discussed in detail.

Consider that two SUs, denoted by $A$ and $B$, sequentially run through the $T$ positions in Figure 1 in opposite directions (i.e., SU $A$ runs clockwise and SU $B$ runs anticlockwise, for instance), one position at a time with the same speed. Then, the $\mathrm{CH}$ sequences can be described as:

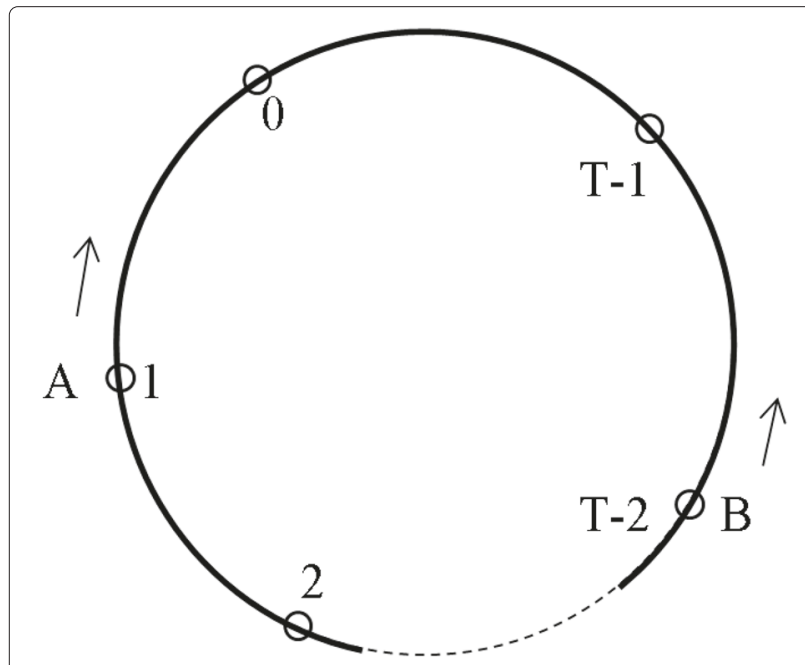

Figure 1 Channel distribution.

$$
\begin{aligned}
& S_{A}(t)=\left(S_{A}(0)-t\right) \bmod T, \\
& S_{B}(t)=\left(S_{B}(0)+t\right) \bmod T,
\end{aligned}
$$

where $S_{A}(0)$ and $S_{B}(0)$ are the initial positions in the ring for SUs $A$ and $B$, respectively, and $a \bmod b$ is the non-negative remainder of the division of $a$ by $b$.

Let $d(t): 0 \leq d(t)<T$ be the distance between the SUs which can be computed as a number of nodes between SUs $A$ and $B$ at time slot $t$, such that:

$$
d(t)= \begin{cases}S_{A}(t)-S_{B}(t), & S_{A}(t) \geq S_{B}(t) \\ T+S_{A}(t)-S_{B}(t), & S_{A}(t)<S_{B}(t)\end{cases}
$$

Theorem 1. If all channels are available for the SUs and $d(0)$ is even, the rendezvous occurs in $t_{r}=\frac{d(0)}{2}$ time slots.

Proof. For the rendezvous to occur at time slot $t_{r}=$ $\frac{d(0)}{2}$, we must have $S_{A}\left(t_{r}\right)=S_{B}\left(t_{r}\right)$. If $S_{A}(0) \geq S_{B}(0)$, from (3) we have that $S_{B}(0)=S_{A}(0)-d(0)$. Then, with the help of (2)

$$
\begin{aligned}
S_{B}\left(t_{r}\right) & =\left(S_{A}(0)-d(0)+\frac{d(0)}{2}\right) \bmod T \\
& =\left(S_{A}(0)-\frac{d(0)}{2}\right) \bmod T=S_{A}\left(t_{r}\right) .
\end{aligned}
$$

If $S_{A}(0)<S_{B}(0)$, then from (3) $S_{B}(0)=T+S_{A}(0)-d(0)$, and consequently

$$
\begin{aligned}
S_{B}\left(t_{r}\right) & =\left(T+S_{A}(0)-d(0)+\frac{d(0)}{2}\right) \bmod T \\
& =\left(T+S_{A}(0)-\frac{d(0)}{2}\right) \bmod T=S_{A}\left(t_{r}\right) .
\end{aligned}
$$

Alternatively, $S_{A}\left(t_{r}\right)=S_{B}\left(t_{r}\right)$ implies $d\left(t_{r}\right)=0$. By noting that in each time slot both SUs sequentially jump in opposite directions (see Figure 1 ), if $d(t) \neq 1$ then in the next time slot the distance between them decreases by 2 , so that $d(t+1)=d(t)-2$ (i.e., the distance between SUs decreases twice the number of elapsed time slots). Therefore, the time to rendezvous can be calculated as $t_{r}=\frac{d(0)}{2}$ for $d(0)$ even.

Following the above reasoning, it is easy to see that, if $d(0)$ is odd, after $\frac{d(0)+1}{2}$ time slots the SUs transpose their positions without meeting each other. Once the SUs transpose their positions, the new resulting distance is $T-1$. Now, if $T$ is even, the new distance, just after the SUs transpose their positions, is odd and the SUs indefinitely run through the ring without ever meeting. To overcome this problem, in what follows we restrict the analysis by making $T$ odd.

Theorem 2. If all channels are available for the SUs and $d(0)$ and $T$ are odd, rendezvous occurs in $t_{r}=\frac{d(0)+T}{2}$ time slots. 
Proof. Given that $d(0)$ is odd, from the above discussion, we have $d\left(\frac{d(0)+1}{2}\right)=T-1$. Since $T$ is odd, $T-1$ is even and from Theorem 1 we can write $d\left(\frac{d(0)+1}{2}+\frac{T-1}{2}\right)=$ 0 which implies $t_{r}=\frac{d(0)+T}{2}$.

\subsection{Rendezvous diversity analysis}

To compute the rendezvous diversity of the $\mathrm{CH}$ sequences presented in Equations 1 and 2, let us recall the example in Figure 1, where it can be seen that the rendezvous occurs in a single channel on every lap around the ring. Also given that Equations 1 and 2 exhibit a periodic behavior with period $T$ and that $d(t)$ is a linear combination of $S_{A}(t)$ and $S_{B}(t)$ we have $d(0)=d(k T), k \in \mathbb{N}^{*}$, where the value of $k$ identifies the number of turns of each $\mathrm{SU}$ around the ring (thus $k=0$ identifies the first round, $k=1$ the second one and so on). This means that the rendezvous channel is the same in all sequences periods and therefore this strategy does not guarantee maximum rendezvous diversity. Therefore, next we discuss the proposed algorithm which aims at maximizing the rendezvous diversity while minimizing the TTR.

\section{Proposed algorithm}

As proved in Theorems 1 and 2 and according to the rendezvous diversity index definition, in order to guarantee finite MTTR and maximize the rendezvous diversity, two conditions must be met: (i) $T$ must be odd and (ii) $|C|=G$. With this in mind we make

$$
T= \begin{cases}N, & \text { for } N \text { odd } \\ N+1, & \text { for } N \text { even }\end{cases}
$$

Note that, in order to make $T$ odd, in the case of $N$ even an additional node is placed in the ring of Figure 1 so that one of the channels is listed twice. We consider important to highlight that even when the authors in [19] also use a ring channel distribution, they restrict their analysis for the case of $N$ even (i.e., the number of networks channels must be even). Also, the construction of the sequences presented in this section differ greatly from that of the sequences described in [19].

In what follows we propose the full diversity channel hopping algorithm with two variants, one that is role based (FDCH-RB) and another that is a common strategy (FDCH-CS). We first present the role based rendezvous algorithm and, as in [15,17-19], we assume that the SUs attempting to rendezvous have different preassigned roles which are allocated before the rendezvous process starts. In the sequel, we use the terms transmitter and receiver in order to differentiate the role of each SU. In our proposal, the transmitter runs clockwise and jumps in each time slot. The receiver runs anticlockwise and jumps for $T-1$ time slots but remains at the last node for one additional time slot before starting to jump for the next $T-1$ time slots. This behavior of the receiver ensures maximum rendezvous diversity as we will discuss in Section 5.2.

Moreover, we consider that each sequence replaces the unavailable channels by an available one. Then, let $S_{T}(t)$ and $S_{R}(t)$ be the $\mathrm{CH}$ sequences of the transmitter and the receiver, respectively. The procedure for sequences construction are shown in Algorithms 1 and 2 where $S_{T}(0)$, $S_{R}(0)$ denote the initial channels and $A_{T}, A_{R}$ are the set of available channels of the transmitter and the receiver, respectively.

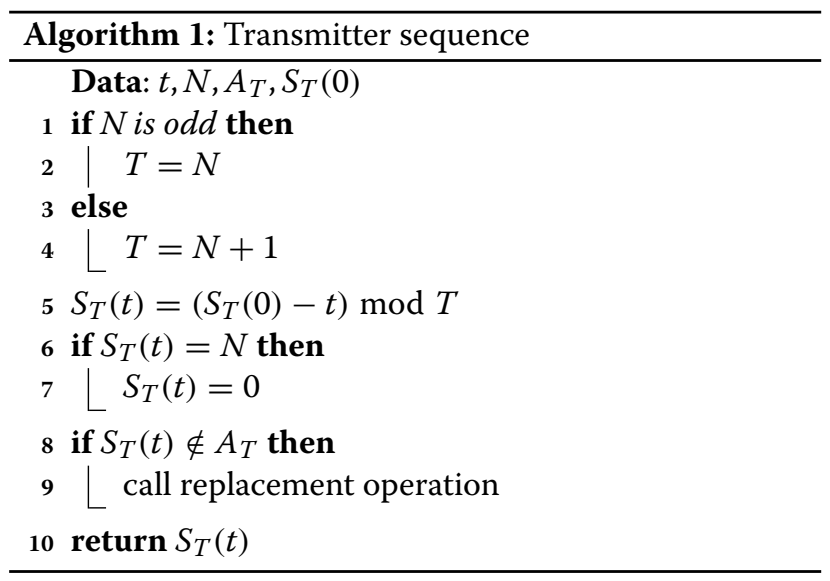

An example of sequences for even and odd values of $N$ is shown in Figure 2. Due to the lack of synchronization between SUs, each one can begin its $\mathrm{CH}$ sequence at different time slots. The time to rendezvous is measured taking as starting point the time slot where both sequences have already begun. In Figure 2a, $N=4$ and the additional channel is marked as 4(0). The first number is used to measure the distance between SUs and the number in parentheses is the actual channel selected in the $\mathrm{CH}$ 


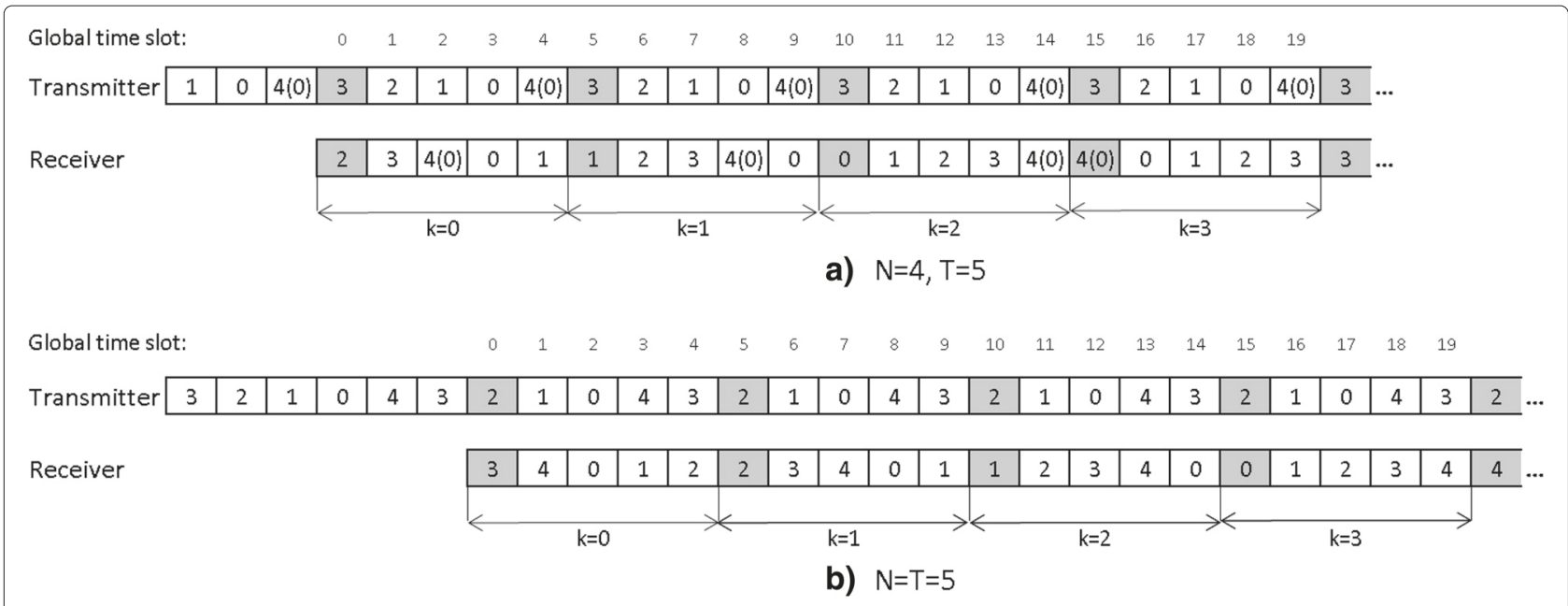

Figure 2 Transmitter and receiver sequences. (a) $N$ is even; (b) $N$ is odd.

sequence. Since both SUs use the same additional channel in every turn around the ring, rendezvous takes place.

\subsection{ETTR and MTTR performance}

By using the mathematical framework presented in [28], the ETTR can be computed as:

$$
\operatorname{ETTR}=\frac{1}{T} \sum_{d(0)=0}^{T-1} \min \left\{t_{r}: S_{T}\left(t_{r}\right)=S_{R}\left(t_{r}\right)\right\}
$$

By Theorems 1 and 2, the sequences intersect for first time after

$$
t_{r}= \begin{cases}\frac{d(0)}{2}, & \text { for } d(0) \text { even } \\ \frac{d(0)+T}{2}, & \text { for } d(0) \text { odd }\end{cases}
$$

time slots. Therefore, (7) can be rewritten as:

$$
\begin{aligned}
\text { ETTR } & =\frac{1}{T}\left\{\sum_{d(0)=1}^{\frac{T-1}{2}} \frac{2 d(0)}{2}+\sum_{d(0)=1}^{\frac{T-1}{2}}\left[\frac{2 d(0)-1+T}{2}\right]\right\} \\
& =\frac{1}{T}\left\{2 \sum_{d(0)=1}^{\frac{T-1}{2}} d(0)+\sum_{d(0)=1}^{\frac{T-1}{2}} \frac{T-1}{2}\right\} \\
& =\frac{T-1}{2}
\end{aligned}
$$

Additionally, from (8) the maximum time to rendezvous occurs when $d(0)$ is odd and equal to $T-2$, then by making $d(0)=T-2$ in (8) we have MTTR $=T-1$. The greatest value of MTTR for the proposed FDCH-RB algorithm happens when $T=N+1$, in such case $\mathrm{MTTR}=N$. Note that this value is comparable with the MTTR for the SRB algorithm and smaller than that of the MTTR for the
A-MOCH and ARCH algorithms (see Table 1). We consider important to highlight that the ETTR and MTTR as computed by the above equations are valid when all $N$ channels are available for the SUs. In the next section, we analyze the MCTTR as the maximum TTR in scenarios where the number of common available channels is less than $N$.

\subsection{Rendezvous diversity and MCTTR}

As described at the beginning of Section 5 and shown in Figure $2 b$, at every time slot, the transmitter jumps to the next position in the sequence. However, the receiver follows the jump pattern for $T-1$ time slots and then stays in the same channel for one time slot before the new jump period. As a consequence, the distance, as defined in (3), between SUs at the beginning of each lap around the ring increases by one with respect to the previous lap, except when the distance has its maximum value in which case is reduced to zero, that is

$$
d((k+1) T)=(d(k T)+1) \bmod T
$$

and by solving the recurrence relation (10) we obtain

$$
d(k T)=(d(0)+k) \bmod T .
$$

Note that $d(k T)$ takes values in the range from 0 to $T-1$ being an infinite sequence of truncated discrete ramp functions concatenated together. Then, after $T$ laps of the SUs around the ring (i.e., from $k=0$ to $k=T-1$ ), $d(k T)$ has swept all possible integer values in its output range. Also, it is important to highlight that the proposed algorithm guarantees rendezvous for each lap around the ring. Let $t_{r}^{k}$ be the TTR in the $k$ th lap around the ring, 
measured from the beginning of that lap. Then, using (8) and (11), $t_{r}^{k}$ can be written as

$$
t_{r}^{k}= \begin{cases}\frac{d(k T)}{2}, & \text { for } d(k T) \text { even } \\ \frac{d(k T)+T}{2}, & \text { for } d(k T) \text { odd. }\end{cases}
$$

By evaluating the transmitter sequence in $t_{r}^{k}$ we have

$$
S_{T}\left(t_{r}^{k}\right)=\left(S_{T}(0)-t_{r}^{k}\right) \bmod T .
$$

Note that each different value of $d(k T)$ implies a different value of $t_{r}^{k}$ in the range from 0 to $T-1$. Hence, after $T$ turns around the ring, the rendezvous will have occurred in all possible channels according to (13). In other words, in every lap around the ring, the initial distance between SUs changes, modifying the next time to rendezvous and the resulting rendezvous channel. This means that FDCH-RB guarantees maximum rendezvous diversity.

Theorem 3. The MCTTR for the FDCH-RB algorithm is $M C T T R=T^{2}-1$.

Proof. If not all channels are available for the SUs, due to the maximum rendezvous diversity property, in the worst case the rendezvous occurs in lap $k=T-1$. By noting that at the start of lap $k$, the elapsed time since the beginning of the rendezvous process is $k T$, the maximum conditional TTR (i.e., for the worst case) can be computed as MCTTR $=(T-1) T+M T T R$. Since, as derived in Section 5.1, in a lap where rendezvous is guaranteed the MTTR is $T-1$, we have MCTTR $=T^{2}-1$.

The greatest value of MCTTR happens when $N$ is even, in such case $T=N+1$ and MCTTR $=N^{2}+2 N$. This value is greater than that of the MCTTR for A-MOCH and ARCH algorithms. However, for $N$ odd the MCTTR of the proposed FDCH-RB algorithm is MCTTR $=N^{2}-1$ which is slightly smaller than the MCTTR for the A$\mathrm{MOCH}$ algorithm. Note that the ARCH algorithm only ensures rendezvous when $N$ is even (see Table 1).

Additionally, the jump pattern of the transmitter causes that the channel $S_{T}(t+k T)$ is the same for any value of $k$. However, as a consequence of the jump-stay pattern of the receiver, $S_{R}(t+k T)$ sweeps all possible values in its output range for $0 \leq k \leq T-1$. Then, in each lap around the ring to the same channel $S_{T}(t+k T)$, corresponds a different channel $S_{R}(t+k T)$ (e.g., time slots 1,6 , 11, and 16 in Figure 2a). Exploiting this feature, if the SUs are under the symmetric model, the unavailable channels are replaced by one channel in $A_{u}$ (i.e., the set of available channels seen by $\mathrm{SU} u$ ) in the following $T$ laps to the ring as described in line 2 of Algorithm 3, where the notation $A u(i)$ is used to indicate the $i$ th element of $A u$. Note that for the symmetric model $G=\left|A_{u}\right|$ and SUs always replace unavailable channels by a commonly available one.

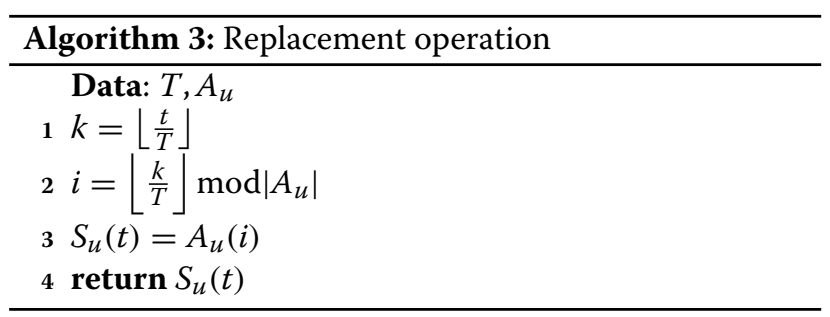

However, under the asymmetric model, $G \neq\left|A_{u}\right|$ and therefore, the replacement operation described above offers no advantage. In this case, we propose a random replacement operation. Note that since the proposed strategy ensures maximum rendezvous diversity, the random nature of the replacement operation do not compromise the MCTTR. The random replacement operation is described in Algorithm 4.

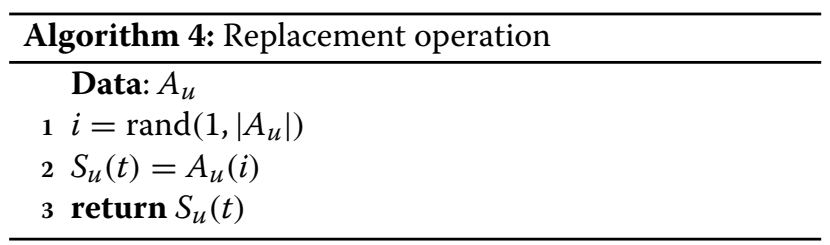

\subsection{Removing the preassigned roles}

The $\mathrm{CH}$ sequences construction described in the previous section gume slots, the ETTR can be computed as:arantees rendezvous and ensures maximum rendezvous diversity but requires that the roles be preassigned, which may not be possible in all scenarios. To overcome this drawback, the authors in [17] propose that each SU uses its ID to alternate between the role of transmitter and receiver. Although this proposal guarantees rendezvous, the performance in terms of ETTR of the CS strategy is worse than the RB strategy.

To solve the problem of allocation of roles, we equipped the SUs with two radios that operate simultaneously. One radio follows the transmitter sequence and the other the receiver sequence. Since the SUs implement the same algorithms, they perform a CS strategy. The appropriate use of more than one radio improves the ETTR and MTTR as was concluded in [29] and as we are going to show in the sequel. Figure 3 shows the basic idea behind our maximum diversity channel hopping common strategy (FDCH-CS) algorithm. Let $S_{T u}(t)$ and $S_{R u}(t)$ denote the transmitting and receiving $\mathrm{CH}$ sequences followed by the $\mathrm{SU} u$, and $S_{u}(0)$ the initial position of $\mathrm{SU} u$ on the ring. 


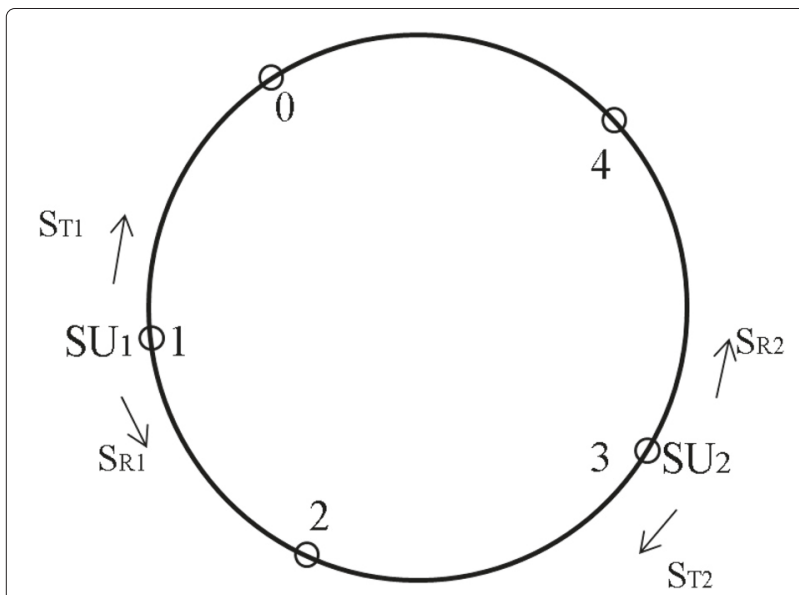

Figure 3 Common strategy. $N=5, d_{12}(0)=3, d_{21}(0)=2$.

Since each SU runs both sequences simultaneously, the rendezvous is expected to be achieved between the transmitter sequence of one $S U$ and the receiver sequence of the other (i.e., $S_{T 1}(t)=S_{R 2}(t)$ or $S_{T 2}(t)=S_{R 1}(t)$ ). Then, there exist two different values of $d(0): d_{12}(0)$ for $S_{T 1}(0)$ with respect to $S_{R 2}(0)$ and $d_{21}(0)$ for $S_{T 2}(0)$ with respect to $S_{R 1}(0)$. Algorithm 5 shows the proposed common strategy (FDCH-CS).

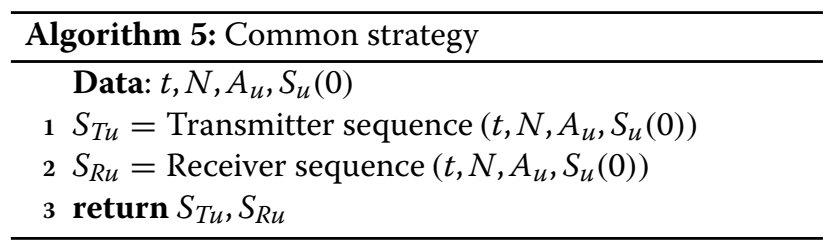

Theorem 4. If two SUs use the FDCH-CS algorithm to generate their $C H$ sequences, then: $M T T R=\frac{T-1}{2}$ and $E T T R=\frac{(T-1)(T+1)}{4 T}$.

Proof. Considering that the SUs begin the rendezvous process at the same time and from Figure 3, it can be noted that $d_{12}(0)+d_{21}(0)=T$. Given that $T$ is odd, always one of the distance values between SUs $\left(d_{12}(0)\right.$ or $\left.d_{21}(0)\right)$ is even. We refer to this value as $d_{p}(0)$. Note that even when the SUs do not begin the rendezvous process at the same time, $0 \leq d_{p}(0) \leq T-1$, so that the worse case occurs when the SUs begin the rendezvous process at the same time and the maximum value of $d_{p}(0)$ is $T-1$ and by Theorem 1 MTTR $=\frac{T-1}{2}$.

In Figure $3 d_{21}(0)=d_{p}(0)=2$, however, if $\mathrm{SU}_{2}$ was at node 4 in the ring, then $d_{12}(0)=2, d_{21}(0)=3$ and also $d_{p}(0)=2$. This analysis leads to a value of $d_{p}(0)$ that is the same for two different positions of $\mathrm{SU}_{2}$ in the ring. Then, considering that $d_{p}(0)=0,2,4, \ldots T-1$, the probability of $d_{p}(0)$ assuming a given value is

$$
\operatorname{Pr}\left[d_{p}(0)\right]= \begin{cases}\frac{1}{T}, & \text { for } d_{p}(0)=0 \\ \frac{2}{T}, & \text { for } d_{p}(0)>0 \text { and } d_{p} \text { even. }\end{cases}
$$

As the SUs intersect for the first time after $t_{r}=\frac{d_{p}(0)}{2}$ time slots, the ETTR can be computed as:

$$
\begin{aligned}
\text { ETTR } & =\frac{2}{T} \sum_{d_{p}(0)=1}^{\frac{T-1}{2}} \frac{2 d_{p}(0)}{2} \\
& =\frac{1}{T}\left[\left(\frac{T-1}{2}\right)\left(\frac{T-1}{2}+1\right)\right] \\
& =\frac{(T-1)(T+1)}{4 T} .
\end{aligned}
$$

Theorem 5. If two SUs use the FDCH-CS algorithm to generate their CH sequences, then $M C T T R=T^{2}-1$.

Proof. Because the SUs follow $S_{T}(t)$ and $S_{R}(t)$ sequences simultaneously, they meet twice in every lap around the ring. As we also considered in the proof of Theorem 4, the rendezvous happens for the first time at $t_{r}=\frac{d_{p}(0)}{2}$. Furthermore, there is a distance value between SUs that is odd $\left(T-d_{p}(0)\right)$. Considering the second term of (8), the SUs rendezvous for the second time at $t_{s}=\frac{2 T-d_{p}(0)}{2}$.

The rendezvous channels can be identified as: $c_{1}=$ $S_{T}\left(t_{r}\right)$ and $c_{2}=S_{R}\left(t_{s}\right)$ for the first and second rendezvous, respectively. With the help of Algorithms 1 and 2, $c_{1}$ and $c_{2}$ can be rewritten as:

$$
\begin{aligned}
c_{1} & =\left(S_{T}(0)-\frac{d_{p}(0)}{2}\right) \bmod T, \\
c_{2} & =\left(S_{R}(0)+\frac{2 T-d_{p}(0)}{2}\right) \bmod T \\
& =\left(T+S_{R}(0)-\frac{d_{p}(0)}{2}\right) \bmod T .
\end{aligned}
$$

Considering that the remainder operation is nonnegative and that $S_{T u}(0)=S_{R u}(0)=S_{u}(0)$ it can be seen that $c_{2}=c_{1}$. Then, although SUs meet twice at every lap around the ring, the rendezvous happens in the same channel and according to the proof of Theorem 3 MCTTR $=T^{2}-1$.

Let us remark that in our CS strategy each user follows the $S_{T}$ and $S_{R}$ sequences simultaneously; therefore, as in the $\mathrm{RB}$ strategy, maximum rendezvous diversity is also guaranteed. Also we consider important to highlight that the FDCH-CS algorithm, in terms of ETTR and MTTR, 
performs better than the FDCH-RB algorithm. This is an expected result due to the fact that FDCH-CS uses two radios.

\section{Multi-user rendezvous algorithm}

The proposed FDCH-CS algorithm can be extended to a multi-user scenario. The purpose is to achieve rendezvous for $M$ SUs by multiple pair-wise rendezvous. Once two SUs rendezvous in one of the channels, they interchange information to synchronize their parameters and, after that, the two SUs generate an identical $\mathrm{CH}$ sequence.

Each SU $u$ has two parameters to determine the jump sequence: the initial channel $\left(S_{u}(0)\right)$ and the current time slot since the beginning of its sequence $\left(t_{u}\right)$. After $\mathrm{SU}_{1}$ and $\mathrm{SU}_{2}$ successfully rendezvous, each one updates its parameters according to the following strategy: $S_{1}(0)=S_{2}(0)=$ $\min \left\{S_{1}(0), S_{2}(0)\right\}$ and $t_{1}=t_{2}=\min \left\{t_{1}, t_{2}\right\}$. Afterward, the two SUs generate an identical $\mathrm{CH}$ sequence.

As an illustrative example, consider the scenario shown in Figure 4 where $P_{u}$ is the parameter set of $\mathrm{SU}_{u}$ and the lines between users denote that they are within the communication range of each other (i.e., they are neighbors). Since two users may have different available channels, the replacement operation described in Section 5.2 may cause that each user replaces unavailable channels by a different one. This replacement operation may broken the previous parameter synchronization.

To illustrate this phenomenon, let us consider that $\mathrm{SU}_{1}$ and $\mathrm{SU}_{2}$ achieve rendezvous and synchronize their parameters, so they visit the commonly available channels in the same time slots. Afterward, if $\mathrm{SU}_{2}$ performs the replacement operation and eventually achieves rendezvous with $\mathrm{SU}_{3}$, the parameters synchronization between $\mathrm{SU}_{2}$ and $\mathrm{SU}_{3}$ may break the previous synchronization between $\mathrm{SU}_{2}$ and $\mathrm{SU}_{1}$. Although the pair-wise rendezvous has been proposed in $[25,26]$, these works do not consider the problem of breaking the previous parameter synchronization. To avoid this problem, let us define $\mathrm{ChS}_{u}$ as the set of potentially channels for parameter synchronization and the beginning of rendezvous process $\mathrm{ChS}_{u}=A_{u}$. When a rendezvous between two SUs occurs, they only synchronize their parameters if the rendezvous channel belongs to $\mathrm{ChS}_{1} \cap \mathrm{ChS}_{2}$. Let us remark that the only purpose of $\mathrm{ChS}_{u}$ is to ensure the parameters' synchronization between all SUs so, like in the two-user scenario, each SU uses its perceive available channels (i.e.,

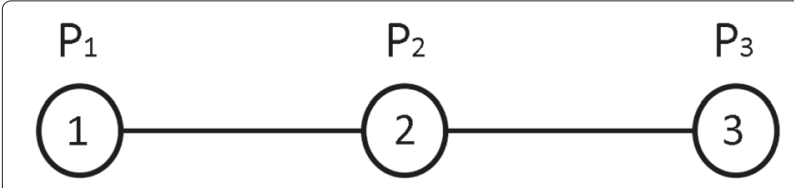

Figure 4 Multi-user scenario.
$\left.A_{u}\right)$ to perform the replacement operation. The proposed multi-user strategy is show in Algorithm 6.

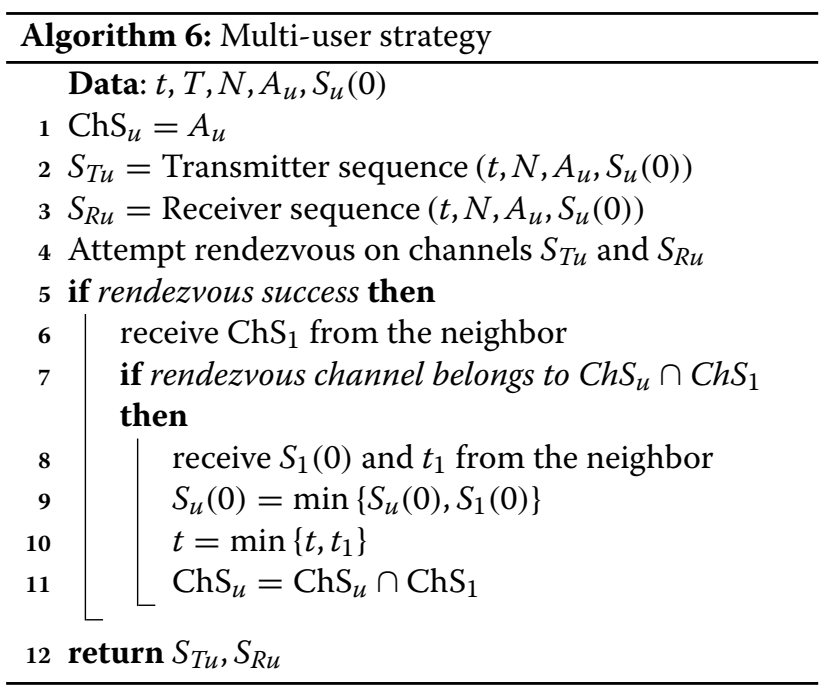

In order to compute the ETTR, MTTR, and MCTTR in the multi-user scenario, let $H: H \in \mathbb{N}$ be the network diameter denoting the maximum number of hops between two SUs (e.g., in Figure 4 the more remote SUs are $\mathrm{SU}_{1}$ and $\mathrm{SU}_{3}$, so $H=2$ ).

Theorem 6. In a CRN with network diameter $H$ and $M \geq 2$ SUs, the MTTR and MCTTR for all SUs to achieve rendezvous are $\operatorname{ETTR}_{M} \leq H\left(\frac{(T-1)(T+1)}{4 T}\right), \operatorname{MTTR}_{M}=$ $H\left(\frac{T-1}{2}\right)$ and $M C T T R_{M}=H\left(T^{2}-1\right)$, respectively.

Proof. Without loss of generality, consider the scenario of Figure 4 with $S_{1}(0)<S_{2}(0)<S_{3}(0)$ and $t_{3}<t_{2}<t_{1}$. In this situation, the three SUs rendezvous when all of them update their parameters to $S_{1}(0)$ and $t_{3}$. For this to happen $H=2$ pair-wise rendezvous are required (i.e., $\mathrm{SU}_{1}$ with $\mathrm{SU}_{2}$ and later, $\mathrm{SU}_{2}$ with $\mathrm{SU}_{3}$ ). Note that independently of the order in which the rendezvous between SUs occurs, always are needed $H$ pair-wise rendezvous. By Theorem 4, in the FDCH-CS strategy, two neighboring SUs rendezvous in at least $\frac{T-1}{2}$ time slots. Then, for any $H$, all SUs in the network rendezvous in at least $\mathrm{MTTR}_{M}=$ $H\left(\frac{T-1}{2}\right)$.

By Theorem 4 the ETTR for two SUs is $\frac{(T-1)(T+1)}{4 T}$. Considering the above analysis and the properties of expected value, then $\operatorname{ETTR}_{M} \leq H\left(\frac{(T-1)(T+1)}{4 T}\right)$. When not all channels are available, two SUs achieve rendezvous in at least MCTTR $=T^{2}-1$ (see Theorem 5). Then, like in the previous analysis, all SUs in the network rendezvous in at least $\mathrm{MCTTR}_{M}=H\left(T^{2}-1\right)$. 


\section{Simulation}

Simulations are used to evaluate the performance of the proposed strategies. The simulations cover two-user and multi-user scenarios under symmetric and asymmetric models. We consider a CRN with at most 45 potentially available channels for SUs $(N \leq 45)$, each SU performs spectrum sensing to determine its set of available channels $\left(A_{u}\right)$. To evaluate the ETTR and the MTTR, we compute the average TTR and the maximum TTR of $10^{5}$ independent runs, respectively. In each run, the SUs are initially placed in a randomly selected channel.

\subsection{Impact of rendezvous diversity}

To evaluate the impact of rendezvous diversity, we assume that all channels are available for the SUs $\left(N=\left|A_{u}\right|=\right.$ $G=45)$. We also consider that in $\alpha N, 0 \leq \alpha \leq 1$ channels, the communication between SUs may fail with a certain probability $\rho$, so that even if a pair of SUs jump to one of these channels at the same time slot, the rendezvous between them may fail ${ }^{\mathrm{c}}$ with probability $\rho$. Table 2 shows the mean of rendezvous diversity index for some of the protocols in Table $1^{\mathrm{d}}$ Note that while RW2, DRSEQ, SSB, and FRCH only guarantee rendezvous in 5\% of the channels, EJS, $\mathrm{ACH}(\mathrm{RB})$, and the proposed schemes ensure maximum rendezvous diversity.

To show the impact of maximum rendezvous diversity, let $\operatorname{ETTR}_{\rho}$ and ETTR 0 be the ETTR for $\rho>0$ and for $\rho=$ 0 , respectively. Thus, we introduce the increased ETTR (IETTR), as a new metric that can be calculated as

$$
\mathrm{I}-\mathrm{ETTR}=\mathrm{ETTR}_{\rho}-\mathrm{ETTR}_{0} .
$$

Figure 5 shows the I-ETTR as a function of $\rho$ when the communications between SUs may fail in $10 \%$ of the channels $(\alpha=0.1)$. It is worth noting that the I-ETTR increases faster with $\rho$ for protocols with $\bar{D}<1$. This result can be extended for $\alpha>0.1$, and although the I-ETTR for all protocols increase, the maximum rendezvous diversity protocols still have better performance, with a smaller increase rate in the I-ETTR. These results show that maximum rendezvous diversity protocols have a certain advantage in realistic scenarios.

Table 2 Mean of rendezvous diversity index $(\bar{D})$

\begin{tabular}{ll}
\hline$\overline{\mathbf{D}}$ & Protocols \\
\hline 0.05 & RW2 [25], DRSEQ [23], SSB [28], and FRCH [16] \\
1.00 & EJS [27], ACH(RB) [17], RPS [29], FDCH-RB, and FDCH-CS
\end{tabular}

RW2, ring walk 2. DRSEQ, deterministic rendezvous sequence. SSB, short sequence-based. $F R C H$, fast rendezvous channel hopping. EJS, enhanced jump stay. ARCH(RB), asynchronous channel hopping (role based). RPS, role-based parallel sequence. $\mathrm{FDCH}-\mathrm{RB}$, full diversity channel hopping- role based. $\mathrm{FDCH}-\mathrm{CS}$, full diversity channel hopping- common strategy.

\subsection{Two-user symmetric scenario}

In this section, we first consider a scenario like the one used in $[25,26,29]$, in which all channels are available for the SUs. Therefore, $N=\left|A_{u}\right|=G=45$, as in the previous section, but only the protocols with the best results in this scenario are included.

Table 3 shows the analytical and simulation results for the selected protocols. Note that we divide the table in four parts. The first one collects the results for DRSEQ, $\mathrm{SSB}$, and FRCH algorithms which use a single radio and the SUs perform a CS strategy to generate identical sequences but, due to the lack of synchronization between SUs, each one starts its $\mathrm{CH}$ sequence at different time slots. In the second part, we include the RW2 and EJS algorithms which use a single radio and the SUs perform a hybrid strategy. In the RW2 algorithm, each SU uses its identification number (i.e., node ID) as a seed to generate the $\mathrm{CH}$ sequence. However, in the EJS algorithm, the seed is a random selected value between one and the smallest prime number greater than or equal to the number of network channels. Next, in the third part, we present the algorithms that also use a single radio but the SUs use a $\mathrm{RB}$ strategy (i.e., the proposed FDCH-RB and $\mathrm{ACH}(\mathrm{RB})$ ). Finally, in the last part, RPS and the proposed FDCH-CS are included. Both of them consider that the SUs have two radios. It is important to note (see Table 1) that in the RPS algorithm, each SU uses a hybrid strategy to generate the $\mathrm{CH}$ sequence. Also, the authors of RPS exploit the use of more than two radios; however, this fact increases the complexity of secondary devices. Hence, we only consider the simpler case in which each SU has two radios.

Note that, although RB strategies in general should outperform the CS and hybrid approaches, the ETTR and MTTR performance of $\mathrm{ACH}(\mathrm{RB})$ is worse than that of EJS, which is an hybrid strategy. Moreover, it is also worth noting that in terms of ETTR and MTTR, the analytical and simulation results of the proposed FDCH-RB algorithm match very well and that FDCH-RB outperforms the $\mathrm{ACH}(\mathrm{RB})$ strategy and also the other strategies. On the other hand, the analytical results for EJS and RW2 provided by their authors are only upper bounds.

In the case of protocols which consider that SUs have two radios, the performance of RPS in terms of ETTR is better than the proposed FDCH-CS. However, in terms of MTTR, the performance of RPS is almost twice worse than the proposed FDCH-CS. Also note that the analytical and simulation results of the proposed FDCH-CS algorithm match very well, while for the RPS algorithm the analytical result for ETTR and MTTR are the same (please see Theorem 4 in [29] for more information about this result).

The scenario described above assumes that the network channels are free of PU activity, but in the context of cognitive radio, this consideration may be unrealistic. In the 


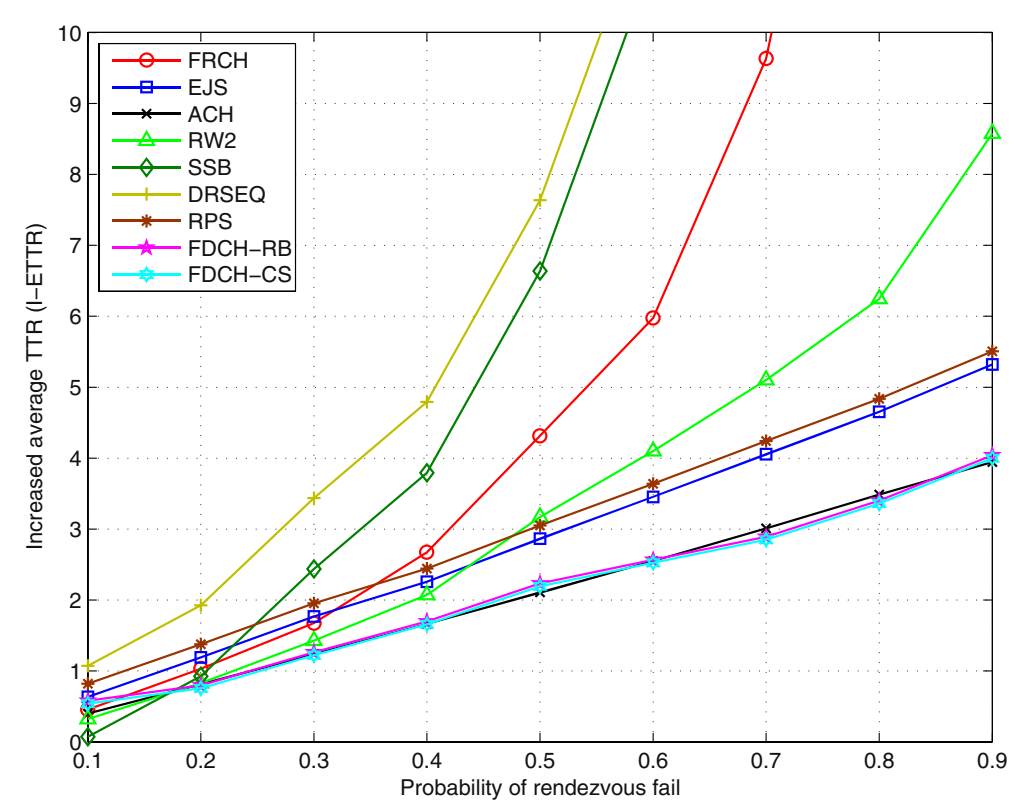

Figure 5 Impact of rendezvous diversity. $N=45$ and $\alpha=0.1$

sequel we consider that the SUs share the same available channels, but not all of them are free of PU activity (i.e., $\left|A_{u}\right|=G$ and $\left.\left|A_{u}\right| \leq N\right)$. Note that this is a typical scenario for a CRN in which SUs are in a small area. Figures 6 and 7 show the ETTR and MTTR results in this scenario for single and two-radio protocols, respectively.

Table 3 Analytical and simulation results for $N=G=45$

\begin{tabular}{|c|c|c|c|c|}
\hline \multirow{2}{*}{ Protocol } & \multicolumn{2}{|c|}{ ETTR } & \multicolumn{2}{|c|}{ MTTR } \\
\hline & Analytical & Simulation & Analytical & Simulation \\
\hline \multicolumn{5}{|l|}{ Common strategies } \\
\hline DRSEQ [23] & - & 44.3 & 91 & 91 \\
\hline SSB [28] & 43.5 & 43.2 & 88 & 88 \\
\hline $\mathrm{FRCH}[16]$ & - & 45.1 & 91 & 91 \\
\hline \multicolumn{5}{|l|}{ Hybrid strategies } \\
\hline RW2 [25] & 52.5 & 44.1 & 88 & 88 \\
\hline EJS [27] & 73.5 & 26.0 & 188 & 142 \\
\hline \multicolumn{5}{|l|}{ Role based strategies } \\
\hline $\mathrm{ACH}(\mathrm{RB})[17]$ & - & 42.7 & - & 185 \\
\hline Proposed FDCH-RB & 22 & 22 & 44 & 44 \\
\hline \multicolumn{5}{|l|}{ Multiple radios } \\
\hline RPS [29] & 47 & 9.9 & 47 & 42 \\
\hline Proposed FDCH-CS & 11.2 & 11.1 & 22 & 22 \\
\hline
\end{tabular}

ETTR, expected time to rendezvous. MTTR, maximum time to rendezvous. DRSEQ, deterministic rendezvous sequence. SSB, short sequence-based. $\mathrm{FRCH}$, fast rendezvous channel hopping. RW2, ring walk 2. EJS, enhanced jump stay. $\mathrm{ARCH}(\mathrm{RB})$, asynchronous channel hopping (role based). $\mathrm{FDCH}-\mathrm{RB}$, full diversity channel hopping- role based. RPS, role-based parallel sequence. FDCH-CS, full diversity channel hopping- common strategy.
In Figure 6, we only consider the protocols that perform the replacement operation and attempt to rendezvous at previously identified available channels. The RW2 algorithm presents better results than the EJS algorithm when $G \leq 14$; however, like FRCH and SSB, its ETTR rapidly grows when the number of common available channels increases. For $G>14$ the EJS performs better than RW2, SSB, and FRCH. The proposed FDCH-RB outperforms the RW2, EJS, FRCH, and SSB protocols. Let us remark that in the proposed FDCH-RB the SUs that attempt to rendezvous need preassigned roles.

In the case of protocols which consider that the SUs have two radios, for $G \leq 42$ the proposed FDCH-CS clearly outperforms the RPS algorithm. For $G>35$ the performance of RPS slightly improves and when almost all of the channels are commonly available to SUs $(42 \leq G \leq$ 45), the performance of the RPS algorithm is better than the proposed FDCH-CS.

Figure 7 shows that the MTTR of the FRCH, the RW2, and the SSB algorithms increases faster than for the others. For $G \geq 12$ the performance of all of them is worse than the EJS algorithm and the proposed RB algorithm (FDCH-RB) performs better than FRCH, RW2, SSB, and EJS. When each SU is equipped with two radios, the MTTR performance of the proposed FDCH-CS is twice better than the RPS algorithm.

\subsection{Two-user asymmetric scenario}

Under the asymmetric model, we consider that each $\mathrm{SU}$ has 22 available channels $\left(\left|A_{u}\right|=22\right)$ and $4 \leq G \leq\left|A_{u}\right|$. In this scenario, each $\mathrm{SU}$ jumps to all available channels 


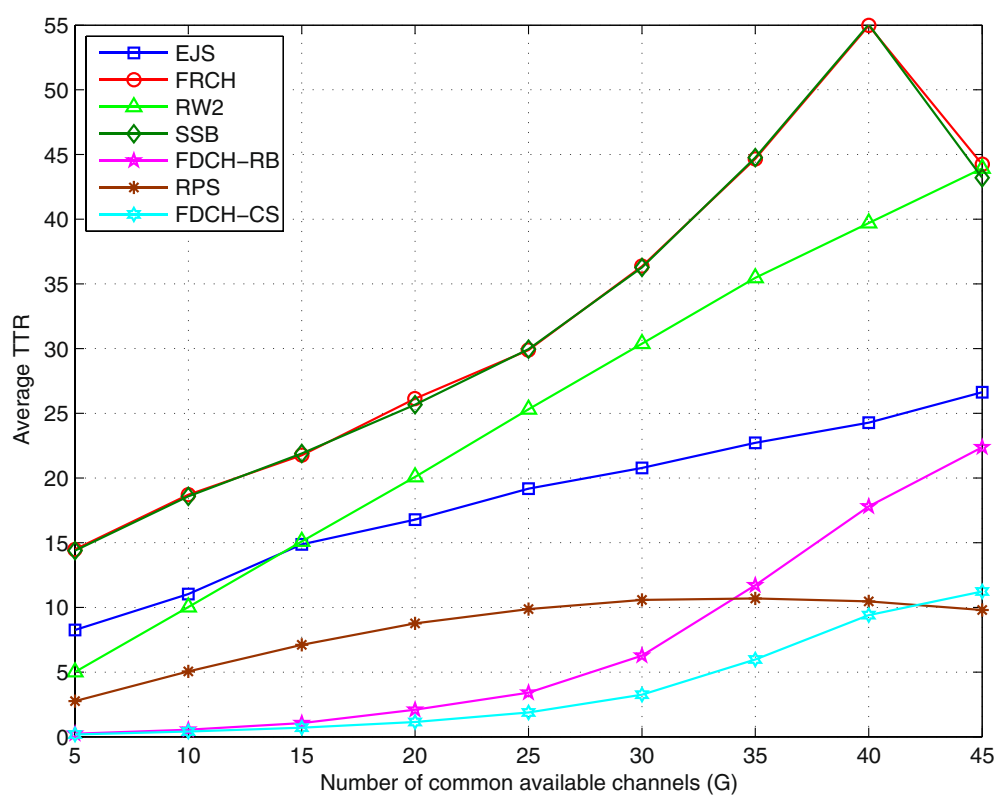

Figure 6 Average TTR for two-user symmetric scenario.

(i.e., all channels in $A_{u}$ ) but can only achieve rendezvous in $G$ channels. The results in Figures 8 and 9 show that for all the compared algorithms the ETTR and MTTR performance improves when the number of common available channels increases.

Figure 8 shows that unlike the symmetric model, the performance of SSB is better than FRCH and EJS, specially for small values of $G$. The performance of RW2 in terms of ETTR is better than FRCH, SSB, and EJS. The proposed FDCH-RB outperforms the other compared protocols. When each SU has two radios, it is worth noting that for $G<16$ the proposed FDCH-CS algorithm outperforms the RPS algorithm. If the number of commonly available channels is close to the amount of available channels seen by each $\mathrm{SU}$ (i.e., $G \approx\left|A_{u}\right|$ ), the performance of RPS and $\mathrm{FDCH}-\mathrm{CS}$ is similar.

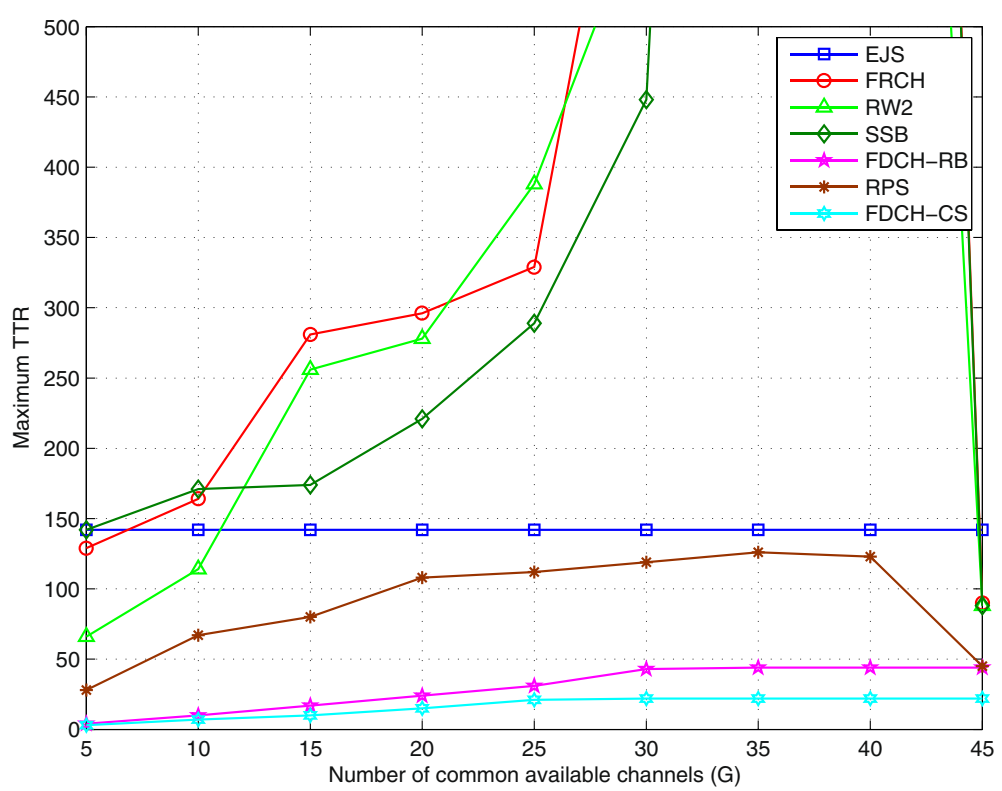

Figure 7 Maximum TTR for two-user symmetric scenario. 


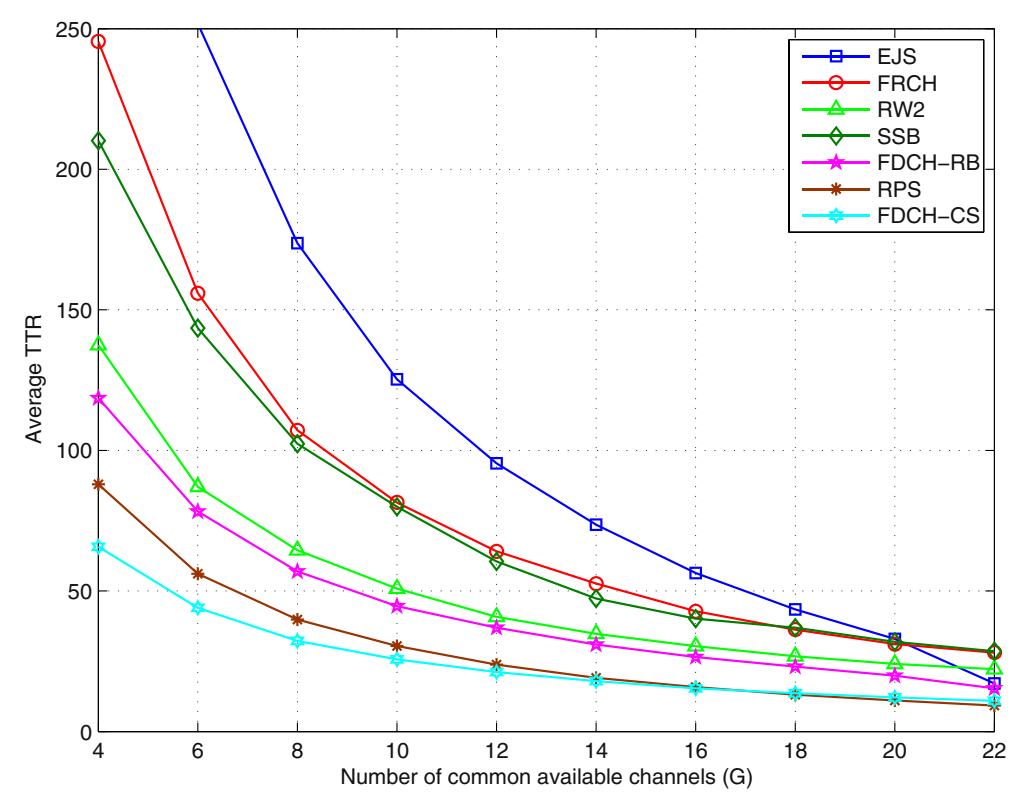

Figure 8 Average TTR for two-user asymmetric scenario.

In terms of MTTR, Figure 9 shows that the EJS algorithm has the worse results among the compared algorithms. The FRCH and SSB algorithms have similar performance and are outperformed by the RW2 algorithm. Again, as was expected, the proposed RB algorithm (FDCH-RB) outperforms the EJS, FRCH, SBB, and RW2 algorithms. When the SUs have two radios, the proposed FDCH-CS performs better than RPS for $G \leq 14$ and for $G>14$ their results are similar.

\subsection{Multi-user scenario}

We compare the proposed FDCH-CS algorithm with RW2 [25] and JS [26] (which were extended to this scenario by their authors). We assume a network with $M=10$ SUs, each one having $\theta N$ available channels. As in $[25,26]$ we set $\theta=0.8$ and consider the symmetric and asymmetric models. For the asymmetric model, we repeatedly generate the available channels for each user until at least one common available channel for all SUs is ensured.

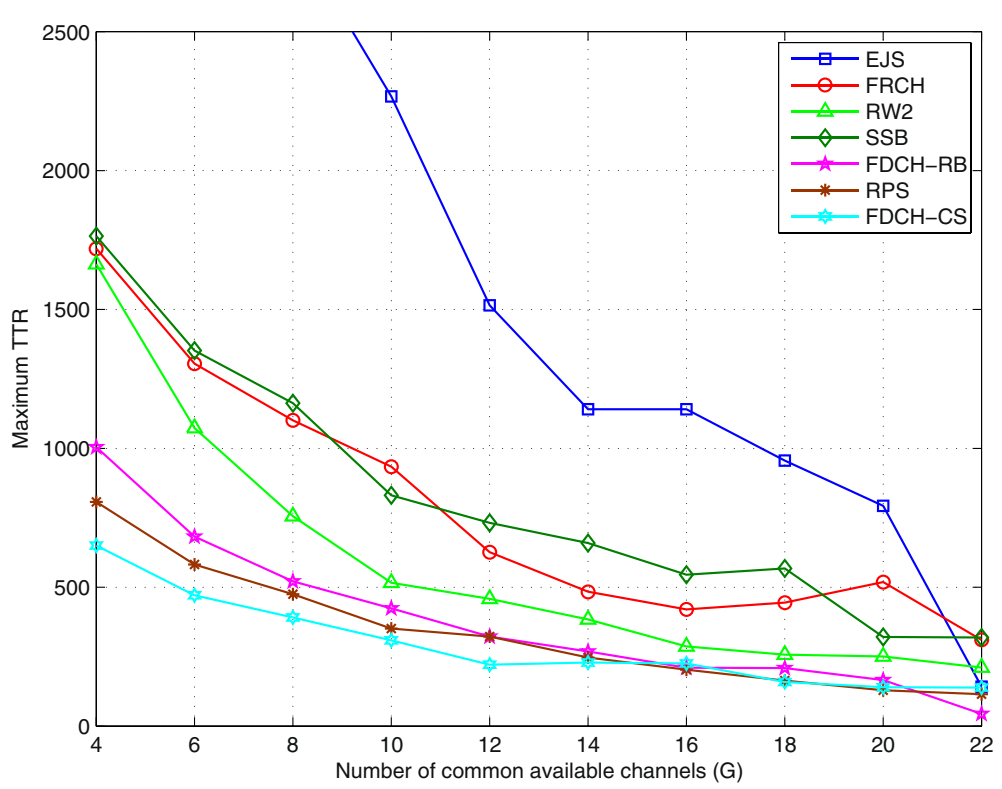

Figure 9 Maximum TTR for two-user asymmetric scenario. 
According to Figure 10, in both models when the number of channels in the network increases, the ETTR for the three algorithms increases. However, in the asymmetric scenario (Figure 10b) the ETTR of the RW2 algorithm grows faster than IS and the proposed FDCH-CS. Note that in the proposed FDCH-CS algorithm each SU has two radios, while in RW2 and JS algorithms each SU has a single radio. Thus, it is reasonable that the proposed FDCH-CS outperforms RW2 and JS in the multi-user scenario.

\section{Conclusions}

In this paper, we focus on the design of $\mathrm{CH}$ sequences that ensure maximum rendezvous diversity and low ETTR for SUs in CRNs. We consider two-user and multi-user rendezvous under symmetric and asymmetric scenarios. Through simulation, we analyze the impact of the rendezvous diversity index in the performance of rendezvous algorithms in CRNs. The results show that the protocols with maximum rendezvous diversity exhibit a better behavior in realistic scenarios.

Two new rendezvous algorithms are proposed, the FDCH-RB and the FDCH-CS. In the first one, the SUs require preassigned roles, while the $\mathrm{FDCH}-\mathrm{CS}$ algorithm removes this constraint by considering that each SU has

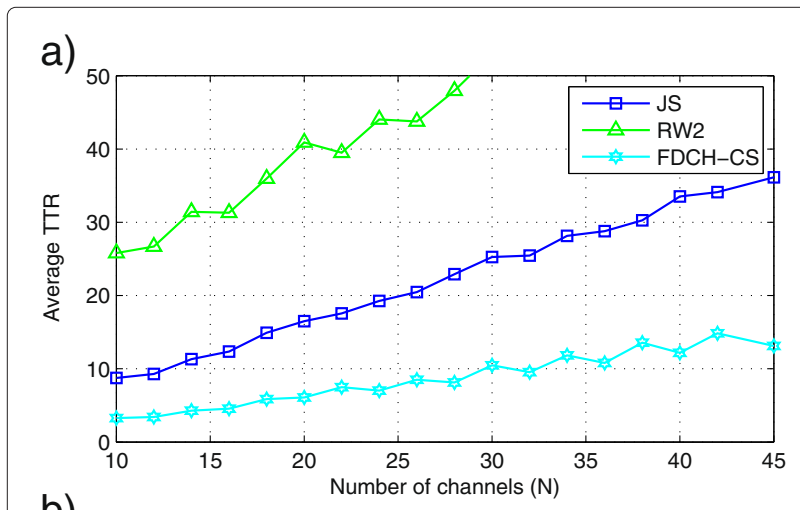

b)

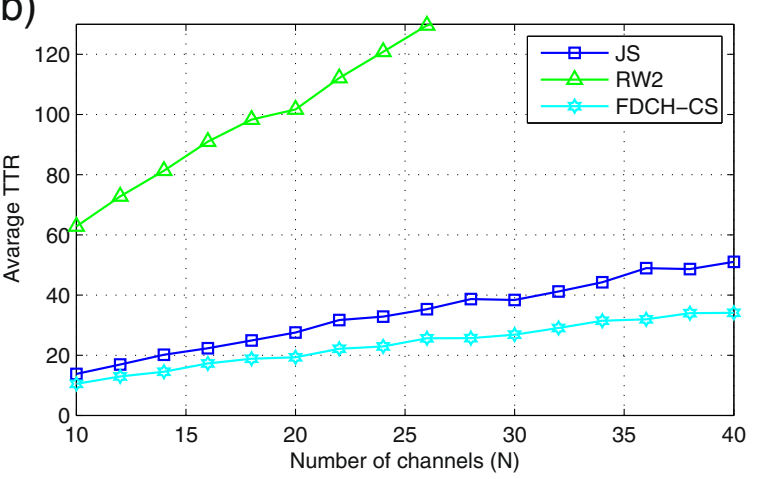

Figure 10 Average TTR for multi-user scenario. $\left|A_{u}\right|=0.8 \mathrm{~N}$ and $M=10$. (a) Symmetric scenario. (b) Asymmetric scenario. two radios, which improves the ETTR and MTTR performance but requires more hardware resources. The theoretical analysis as well as the simulation results show that the proposed algorithms ensure maximum rendezvous diversity and in most scenarios of practical interest outperform other recently proposed schemes.

\section{Endnotes}

${ }^{a}$ How to perform spectrum sensing is out of the scope of this paper. Interesting surveys of spectrum sensing techniques are presented in $[31,32]$

${ }^{\mathrm{b}}$ In this paper, we use the notation $\mathbb{N}^{*}$ to refer to as natural numbers including the zero and $\mathbb{N}$ to refer to as natural numbers without including the zero.

'Even when PUs signals can be perfectly detected, the communication between two SUs may fail because of the hidden transmitter, the exposed transmitter, and the hidden receiver. We refer the reader to [33] for a detailed discussion on these topics.

${ }^{\mathrm{d}}$ The protocols that are not shown in Table 2 have worse results.

\section{Competing interests}

The authors declare that they have no competing interests.

\section{Acknowledgements}

This work was supported by CAPES, CNPq and Araucaria Foundation (Brazil) and MES (Cuba).

\section{Author details}

${ }^{1}$ Central University of Las Villas (UCLV), Camajuaní Highway Km 5 1/2, Santa Clara, Cuba. ${ }^{2}$ Federal University of Technology, Paraná (UTFPR), Avenida Sete de Setembro, Curitiba, Brazil. ${ }^{3}$ Federal University of Paraná (UFPR), Rua XV de Novembro, 1299 - Centro, Curitiba, Brazil. ${ }^{4}$ Pontifical Catholic University of Paraná (PUC), Rua Imaculada Conceicao, 1155 Curitiba, Brazil.

Received: 23 September 2014 Accepted: 9 April 2015

Published online: 09 May 2015

\section{References}

1. IF Akyildiz, W-Y Lee, MC Vuran, S Mohanty, NeXt generation/dynamic spectrum access/cognitive radio wireless networks: a survey. Comput Netw. 50(13), 2127-2159 (2006). doi:10.1016/j.comnet.2006.05.001

2. IF Akyildiz, W-Y Lee, KR Chowdhury, CRAHNs: Cognitive radio ad hoc networks. Ad Hoc Netw. 7(5), 810-836 (2009). doi:10.1016/j.adhoc.2009.01.001

3. LA DaSilva, I Guerreiro, in 2008 3rd IEEE symposium on new frontiers in dynamic spectrum access networks. Sequence-based rendezvous for dynamic spectrum access (Chicago, 2008), pp. 1-7. doi:10.1109/DYSPAN.2008.52

4. Z H Hike, CS Hong, S Lee, The life cycle of the rendezvous problem of cognitive radio ad hoc networks: A Survey. J. Comput. Sci. Eng. 7(2), 81-88 (2013). doi:10.5626/JCSE.2013.7.2.81

5. A Alshamrani, X Shen, L- L Xie, A Cooperative MAC with efficient spectrum sensing algorithm for distributed opportunistic spectrum networks. J. Commun. 4(10), 728-740 (2009)

6. Q Chen, Y-C Liang, M Motani, W-C Wong, A two-level MAC protocol strategy for opportunistic spectrum access in cognitive radio networks. IEEE Trans. Vehicular Technol. 60(5), 2164-2180 (2011). doi:10.1109/TVT.2011.2141694

7. C Cordeiro, K Challapali, D Birru, in First IEEE International symposium on new frontiers in Dynamic Spectrum Access Networks, 2005. DySPAN 2005. IEEE 802.22: the first worldwide wireless standard based on cognitive radios (Baltimore, 2005), pp. 328-337. doi:10.1109/DYSPAN.2005.1542649 
8. L Ma, X Han, C-C Shen, in First IEEE international symposium on new frontiers in Dynamic Spectrum Access Networks, 2005. DySPAN 2005. Dynamic open spectrum sharing MAC protocol for wireless ad hoc networks (Baltimore 2005), pp. 203-213. doi:10.1109/DYSPAN.2005.1542636

9. J Jia, Q Zhang, X Shen, HC-MAC: A hardware-constrained cognitive MAC for efficient spectrum management. IEEE J. Selected Areas Commun. 26(1), 106-117 (2008). doi:10.1109/JSAC.2008.080110

10. C Cormio, KR Chowdhury, A survey on MAC protocols for cognitive radio networks. Ad Hoc Netw. 7(7), 1315-1329 (2009)

11. BF Lo, A survey of common control channel design in cognitive radio networks. Phys. Commun. 4(1), 26-39 (2011). doi:10.1016/j.phycom.2010.12.004

12. J Misic, VB Misic, Probabilistic vs. sequence-based rendezvous in channel-hopping cognitive networks. IEEE Trans. Parallel Distributed Syst. PP(99), 1-1 (2013). doi:10.1109/TPDS.2013.192

13. S Alpern, S Gal, The Theory of Search Games and Rendezvous, 1st edn. (Kluwer Academic, Boston, 2003), p. 319

14. C Cormio, KR Chowdhury, Common control channel design for cognitive radio wireless ad hoc networks using adaptive frequency hopping. Ad Hoc Netw. 8(4), 430-438 (2010). doi:10.1016/j.adhoc.2009.10.004

15. B Kaigui, P Jung-Min, C Ruiliang, Control channel establishment in cognitive radio networks using channel hopping. IEEE J. Selected Areas Commun. 29(4), 689-703 (2011). doi:10.1109/JSAC.2011.110403

16. G-Y Chang, J-F Huang, A fast rendezvous channel-hopping algorithm for cognitive radio networks. IEEE Commun. Lett. 17(7), 1475-1478 (2013). doi:10.1109/LCOMM.2013.060513.130471

17. K Bian, Park J-mJ, Maximizing rendezvous diversity in rendezvous protocols for decentralized cognitive radio networks. IEEE Trans. Mobile Comput. 12(7), 1294-1307 (2013)

18. EO Guerra, VA Reguera, RD Souza, G Brante, EMG Fernandez, Simple role-based rendezvous algorithm for cognitive ad hoc radio networks. Electron. Lett. 50(3), 182-184 (2014). doi:10.1049/el.2013.2994

19. G-Y Chang, W-H Teng, H-Y Chen, J-P Sheu, Novel channel-hopping schemes for cognitive radio networks. IEEE Trans. Mobile Comput., 1-1 (2012). doi:10.1109/TMC.2012.260

20. Y Zhang, Q Li, G Yu, B Wang, in 2011 proceedings IEEE INFOCOM. ETCH: Efficient Channel hopping for communication rendezvous in dynamic spectrum access networks (Shanghai, 2011), pp. 2471-2479. doi:10.1109/INFCOM.2011.5935070

21. Y Zhang, G Yu, Q Li, H Wang, X Zhu, B Wang, Channel-hopping-based communication rendezvous in cognitive radio networks. IEEE/ACM Trans. Netw. PP(99), 1-1 (2013). doi:10.1109/TNET.2013.2270443

22. NC Theis, RW Thomas, LA Dasilva, Rendezvous for cognitive radios. IEEE Trans. Mobile Comput. 10(2), 216-227 (2011). doi:10.1109/TMC.2010.60

23. D Yang, J Shin, C Kim, Deterministic rendezvous scheme in multichannel access networks. Electron. Lett. 46(20), 1402 (2010). doi:10.1049/el.2010.1990

24. J Shin, D Yang, C Kim, A channel rendezvous scheme for cognitive radio networks. IEEE Commun. Lett. 14(10), 954-956 (2010). doi:10.1109/LCOMM.2010.091010.100904

25. H Liu, Z Lin, X Chu, Y-W Leung, in 2010 IEEE/ACM Int'l conference on green computing and communications. Ring-walk based channel-hopping algorithms with guaranteed rendezvous for cognitive radio networks, (2010), pp. 755-760. doi:10.1109/GreenCom-CPSCom.2010.30

26. H Liu, Z Lin, X Chu, Y Leung, Jump-stay rendezvous algorithm for cognitive radio networks. IEEE Trans. Parallel Distributed Syst. 23(10), 1867-1881 (2012)

27. Z Lin, H Liu, X Chu, Y-W Leung, Enhanced jump-stay rendezvous algorithm for cognitive radio networks. IEEE Commun. Lett. 17(9), 1742-1745 (2013). doi:10.1109/LCOMM.2013.071013.131029

28. V Reguera, E Guerra, R Souza, E Fernandez, G Brante, Short channel hopping sequence approach to rendezvous for cognitive networks. Commun. Lett. 18(2), 1-4 (2014). doi:10.1109/LCOMM.2013.112713.132338

29. L Yu, H Liu, Y-W Leung, X Chu, Z Lin, in 2013 IEEE International Conference on Communications (ICC). Multiple radios for effective rendezvous in cognitive radio networks (Budapest, 2013), pp. 2857-2862. doi:10.1109/ICC.2013.6654974
30. K Bian, J-M Park, R Chen, in Proceedings of the 15th annual international conference on Mobile computing and networking-MobiCom '09. A quorumbased framework for establishing control channels in dynamic spectrum access networks, vol. 25 (New York, 2009). doi:10.1145/1614320.1614324

31. T Yucek, H Arslan, A survey of spectrum sensing algorithms for cognitive radio applications. IEEE Commun. Surv. Tutorials. 11(1), 116-130 (2009). doi:10.1109/SURV.2009.090109

32. F Khan, K Nakagawa, in 2013 World Congress on Computer and Information Technology (WCCIT). Comparative study of spectrum sensing techniques in cognitive radio networks (Sousse, 2013), pp. 1-8. doi:10.1109/WCCIT.2013.6618728

33. A De Domenico, E Calvanese Strinati, M-G Di Benedetto, A survey on MAC strategies for cognitive radio networks. IEEE Commun. Surv. Tutorials. 14(1), 21-44 (2012). doi:10.1109/SURV.2011.111510.00108

\section{Submit your manuscript to a SpringerOpen ${ }^{\mathcal{O}}$ journal and benefit from:}

- Convenient online submission

Rigorous peer review

- Immediate publication on acceptance

- Open access: articles freely available online

- High visibility within the field

- Retaining the copyright to your article

Submit your next manuscript at $>$ springeropen.com 